\title{
Further study on oxidation of pseudosapogenins
}

\author{
Izabella Jastrzębska, Krzysztof S. Katryński, and Jacek W. Morzycki* \\ University of Bialystok, Institute of Chemistry, al. Pilsudskiego 11/4, 15-443 Bialystok, Poland \\ E-mail: morzycki@uwb.edu.pl
}

\section{Dedicated to Professor James R. Bull on his retirement}

\begin{abstract}
6 $\beta$-Methoxy-3 $\alpha, 5$-cyclo-5 $\alpha$-furost-20(22)-ene (4) and its 26-tosyloxy derivative (3) were oxidized with $m$-chloroperoxybenzoic acid, dimethyldioxirane and osmium tetroxide. The reaction of 4 with MCPBA yielded the allylic alcohol 6 , the $\alpha, \beta$-unsaturated ketone 7 and the $\alpha$-hydroxy-lactone $\mathbf{8}$. Similar reaction of $\mathbf{3}$ carried out in a buffered medium led to the products 9 and 10 with the $\mathrm{C} 20-\mathrm{C} 22$ bond cleaved. The reaction of the same compound with dimethyldioxirane afforded the allylic alcohol 5. The oxidation of pseudosapogenins with $\mathrm{OsO}_{4}$ gave dihydroxylation products $\mathbf{1 1}$ and $\mathbf{1 2}$.
\end{abstract}

Keywords: Sapogenins, MCPBA, dimethyldioxirane, osmium tetroxide, oxidation, steroids

\section{Introduction}

The majority of medicinally useful steroids are obtained by semi-synthesis from natural products: saponins, phytosterols, cholesterol and bile acids. ${ }^{1-3}$ The immense commercial significance of spirostanol saponins as raw materials for steroid production stems from the discovery by Marker that their aglycones can be easily converted into pregnane derivatives. An excellent historical perspective on the chemistry of Russell Marker was recently described. ${ }^{4}$ The Marker degradation includes acetolysis of the spiroketal moiety followed by an oxidative cleavage of the double bond in the resulting furost-20-enes (pseudosapogenins). The most important sapogenin from an economic standpoint is diosgenin that is extracted from Dioscorea species. Oxidation of the corresponding pseudosapogenin with various reagents, such as $\mathrm{CrO}_{3} / \mathrm{AcOH}, \mathrm{KMnO}_{4} / \mathrm{NaIO}_{4}$ or $\mathrm{H}_{2} \mathrm{O}_{2} / \mathrm{AcOH}$ has been intensively studied. ${ }^{5,6}$ These reactions lead to 16-dehydro-pregnane derivatives by cleavage of the $\mathrm{E}$ ring. They are key intermediates in the synthesis of steroid hormones, particularly corticosteroids and sex hormones.

Some polyhydroxy cholestane derivatives have been recently reported to show interesting biological properties (e.g. cytotoxicity). ${ }^{7-9}$ The aim of this study was to explore the possibility of 
application of spirostanols as starting materials for the synthesis of polyhydroxy cholestane derivatives with the hydroxy groups in the positions 16, 20, 22 and 26.

\section{Results and Discussion}

A conversion of the starting diosgenin 1 into furost-20(22)-enes $\mathbf{3}$ and $\mathbf{4}$ was performed as shown in Scheme 1 and was described in our previous paper. ${ }^{10}$ Therein we present the oxidation of compounds 3 and $\mathbf{4}$ with different reagents ( $m$-chloroperoxybenzoic acid, dimethyldioxirane, and $\left.\mathrm{OsO}_{4}\right)$.

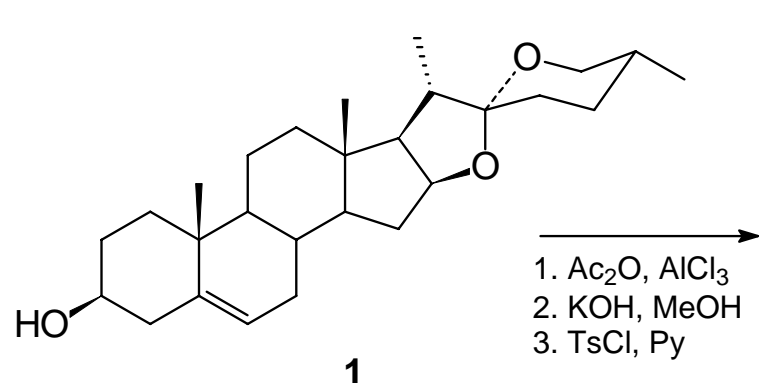

1

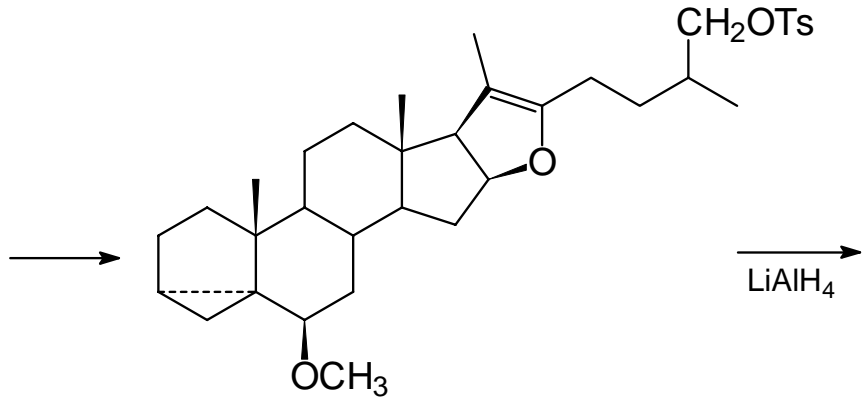

3

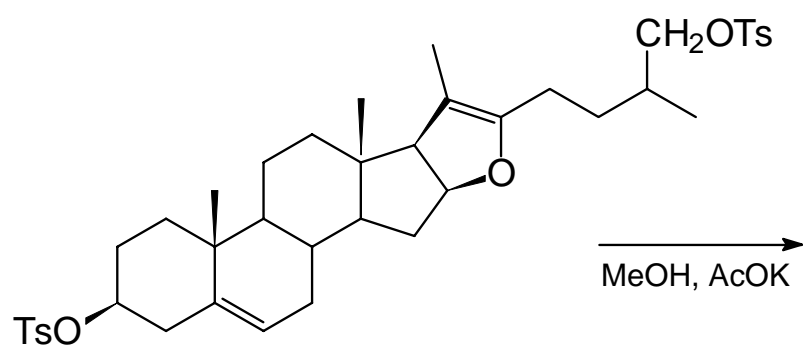

2

\section{Scheme 1}

Compound 4 was treated with MCPBA (Scheme 2). The initial reaction product was presumably the corresponding epoxide, but this proved insufficiently stable under the reaction conditions to be isolated from the reaction mixture. Instead the epoxide readily opened up to yield the allylic alcohol 6 (Z-configuration at the C22-C23 double bond was established by the NOE enhancement of the 21-methyl protons signal on irradiation of 23-H). Further oxidation of 6 with MCPBA gave the $\alpha, \beta$-unsaturated ketone 7. On prolonging the reaction time, the slow disappearance of both products 6 and 7 was observed in favour of the $\alpha$-hydroxy-lactone $\mathbf{8}$. All attempts to isolate the intermediate epoxide under the milder reaction conditions failed. Even MCPBA oxidation performed in a buffered medium $\left(\mathrm{NaHCO}_{3}\right)^{11}$ yielded products derived from 
opening of the intermediate epoxide. For example, the reaction of compound $\mathbf{3}$ afforded esters $\mathbf{9}$ and $\mathbf{1 0}$, as well as the $\alpha$-hydroxy-lactone $\mathbf{8}$. The latter product was identical to the compound obtained from the reaction of 4 with MCPBA. The key step of the reaction carried out in the buffered medium, similarly as in the previous case, was the cleavage of the 20,22-epoxide to the allylic alcohol $\mathbf{5}$. However, further oxidation of $\mathbf{5}$ with excess MCPBA proceeded in a different way in the presence of $\mathrm{NaHCO}_{3}$. The reaction resulted in the cleavage of the $\mathrm{C} 20-\mathrm{C} 22$ bond via a mechanism shown in Scheme 3. In a separate experiment it was found that the hydroxy-tosylate $\mathbf{1 0}$ does not cyclize to $\mathbf{9}$ under the reaction conditions, rather the furan ring closure took place at an earlier stage, presumably by rearrangement of an epoxy-tosylate.

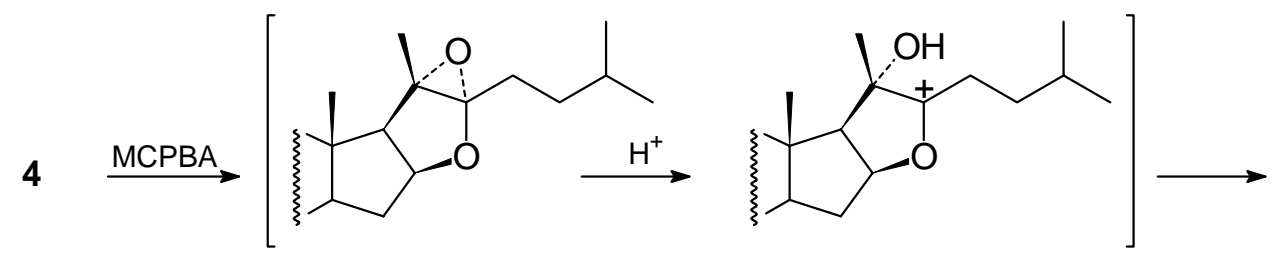<smiles>CC(C)C/C=C1\OC2C[C@H](C(C)C)C(C)(C)C2C1(C)O</smiles><smiles>CC(=O)C1C(OC(=O)[C@H]2C[C@H](C)CO2)CC2C[C@H]3C[C@H]2C13C</smiles>

9<smiles>CC(=O)C1C2CC(C2)CC1OC(=O)[C@@H](O)CC(C)C[SeH2]</smiles>

10

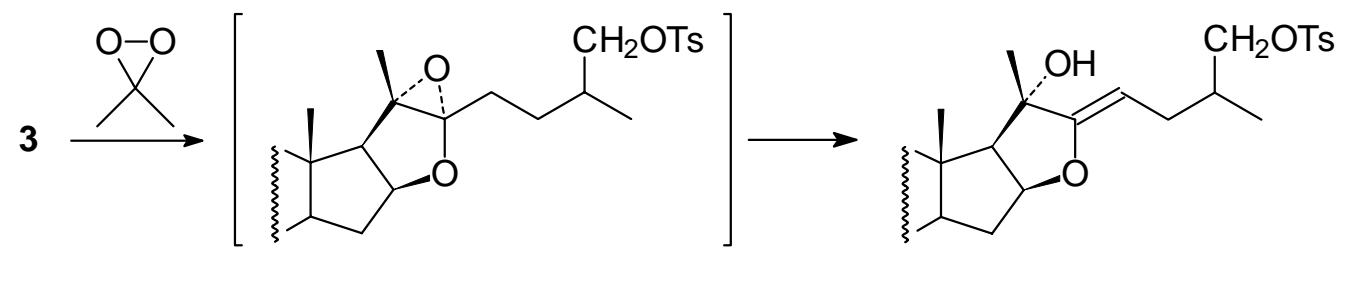

5

Scheme 2 
Since it was postulated ${ }^{8}$ that the 20,22 -epoxide is an intermediate in the enzymatic oxidation of pseudosapogenins, an additional attempt of its synthesis was undertaken. Compound $\mathbf{3}$ was treated with an acetone solution of dimethyldioxirane. ${ }^{12}$ However, this reaction also failed to afford the epoxide, instead the allylic alcohol $\mathbf{5}$ was again isolated as the reaction product.

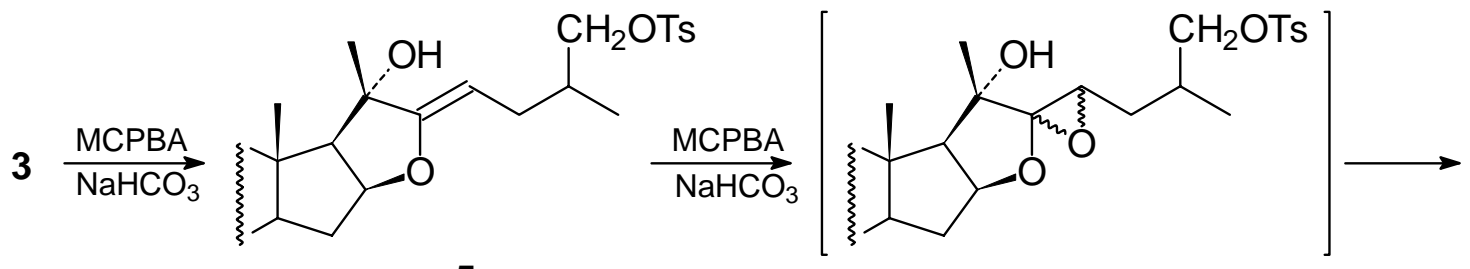

5

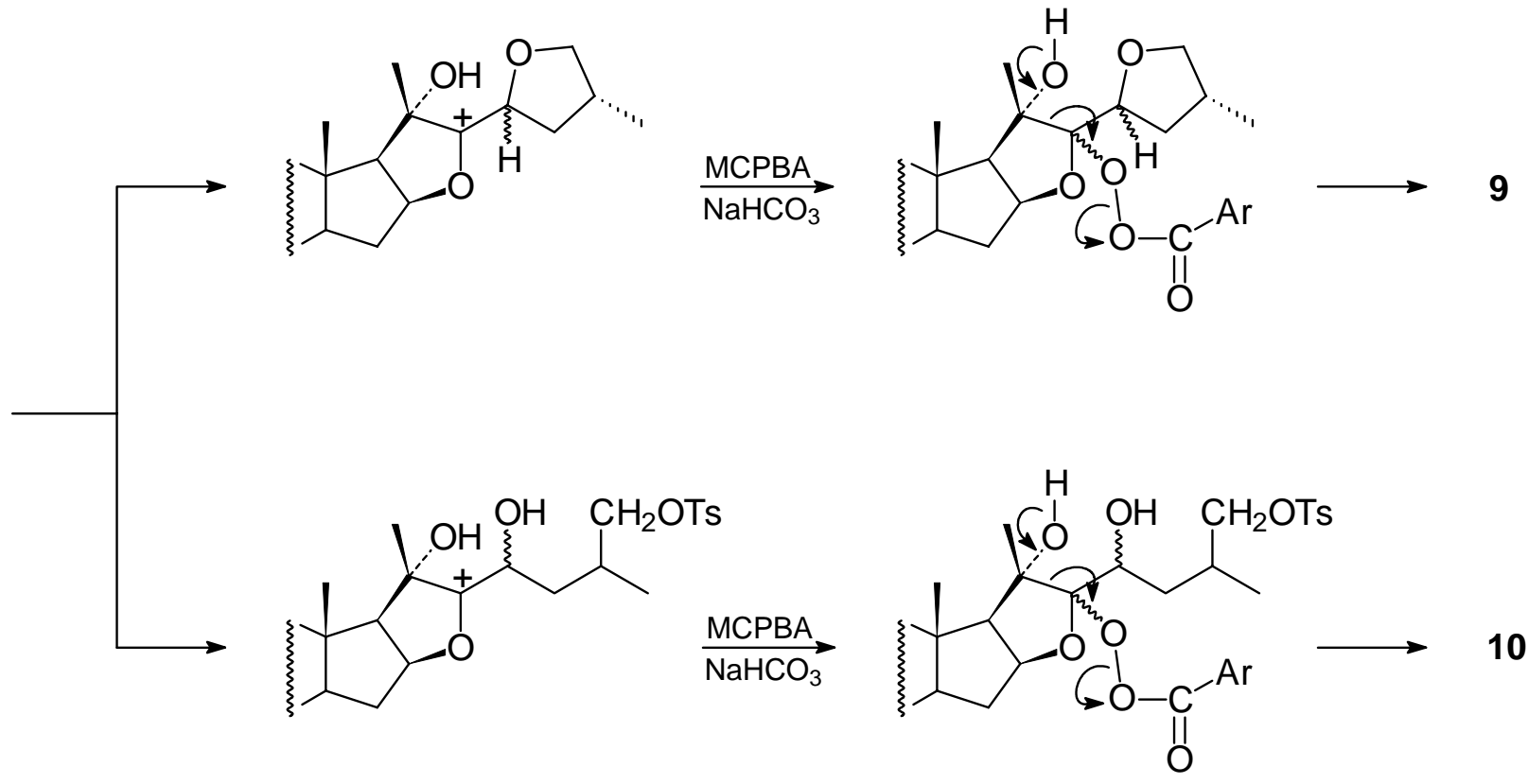

\section{Scheme 3}

In the last series of experiments the oxidation reactions of furost-20(22)-ones $\mathbf{3}$ and $\mathbf{4}$ with osmium tetroxide were studied (Scheme 4). The reaction of $\mathbf{4}$ performed with a stoichiometric amount of reagent afforded the expected product 11. The compound existed in the open-chain form probably stabilized by an intramolecular hydrogen bond. A related product $\mathbf{1 2}$ was obtained when compound 3 was treated with a catalytic amount of $\mathrm{OsO}_{4}$ in the presence of $N$-methylmorpholine $N$-oxide. ${ }^{13}$ The oxidation of the same compound with $\mathrm{OsO}_{4} / \mathrm{H}_{2} \mathrm{O}_{2}$ resulted in the cleavage of the $\mathrm{C} 20-\mathrm{C} 22$ double bond, yielding compound $\mathbf{1 3}$ as the product. Further transformations of compounds $\mathbf{1 1}$ and $\mathbf{1 2}$ are now being studied. 
<smiles>CC(C)CCC(=O)C(C)(O)C1C(O)C[C@@H]2C[C@H]1C2(C)C</smiles>

11<smiles></smiles>

13

\section{Scheme 4}

\section{Experimental Section}

General Procedures. Melting points were determined on a Boetius type Kofler apparatus. NMR spectra were recorded with a Bruker AC $200 \mathrm{~F}$ spectrometer using $\mathrm{CDCl}_{3}$ solutions with TMS as the internal standard (only selected signals in the ${ }^{1} \mathrm{H}$ NMR spectra are reported). Infrared spectra were recorded on a Nicolet series II Magna-IR 550 FT-IR spectrometer as chloroform solutions. Mass spectra were obtained at $70 \mathrm{eV}$ with an AMD-604 spectrometer. The reaction products were isolated by column chromatography performed on 70-230 mesh silica gel (J. T. Baker). TLC was carried out on commercially available plates (Merck, Silica gel $60 \mathrm{~F}_{254}$ ).

Compounds 3 and $\mathbf{4}$ were prepared from diosgenin according to the previously described procedure. $^{10}$ 
Oxidation with dimethyldioxirane. A stirred solution of compound $3(109 \mathrm{mg} ; 0.19 \mathrm{mmol})$ in dichloromethane $(3 \mathrm{~mL})$ was dried with $4 \AA$ molecular sieves and treated with a cold $\left(0{ }^{\circ} \mathrm{C}\right)$ solution of dimethyldioxirane $(0.24 \mathrm{mmol}$ in $4 \mathrm{~mL}$ of acetone). The reaction was continued for 2 hours, filtered and concentrated in vacuo to give an oily residue. Flash chromatography afforded $3 \alpha, 5 \alpha$-Cyclo-6 $\beta$-methoxy-5 $\alpha$-furost-22-ene-20,26-diol 26-monotosylate (5). Eluted with $25 \%$ ethyl acetate/hexane $\left(31.5 \mathrm{mg}, 28 \%\right.$ ) as an amorphous solid. IR, $v_{\max } 3599,3532,1189,1179$, 1098, $909 \mathrm{~cm}^{-1} .{ }^{1} \mathrm{H}$ NMR, $\delta 7.79(2 \mathrm{H}, \mathrm{d}, J=8.0 \mathrm{~Hz}, \mathrm{Ar}), 7.35(2 \mathrm{H}, \mathrm{d}, J=8.0 \mathrm{~Hz}, \mathrm{Ar}), 4.86(1 \mathrm{H}$, $\mathrm{m}, 16 \alpha-\mathrm{H}), 4.19(1 \mathrm{H}, \mathrm{t}, J=7.1 \mathrm{~Hz}, 23-\mathrm{H}), 3.85(2 \mathrm{H}, \mathrm{m}, 26-\mathrm{H}), 3.34\left(3 \mathrm{H}, \mathrm{s}, \mathrm{OCH}_{3}\right), 2.78(1 \mathrm{H}, \mathrm{m}$, $6 \alpha-\mathrm{H}), 2.45\left(3 \mathrm{H}, \mathrm{s}, \mathrm{CH}_{3}-\mathrm{Ar}\right), 1.47(3 \mathrm{H}, \mathrm{s}, 21-\mathrm{H}) ; 1.03(3 \mathrm{H}, \mathrm{s}, 19-\mathrm{H}), 0.89(3 \mathrm{H}, \mathrm{d}, J=6.3 \mathrm{~Hz}, 27-$ $\mathrm{H}), 0.78(3 \mathrm{H}, \mathrm{s}, 18-\mathrm{H}), 0.66(1 \mathrm{H}, \mathrm{m}, 4-\mathrm{H}), 0.45\left(1 \mathrm{H}, \mathrm{dd}, J_{1}=5.1 \mathrm{~Hz}, J_{2}=7.9 \mathrm{~Hz}, 4-\mathrm{H}\right) .{ }^{13} \mathrm{C}$ NMR, $\delta 163.0(\mathrm{C}), 144.5(\mathrm{C}), 133.1(\mathrm{C}), 129.7(2 \times \mathrm{CH}), 127.9(2 \times \mathrm{CH}), 90.6(\mathrm{CH}), 84.2(\mathrm{CH})$, $82.1(\mathrm{CH}), 77.5(\mathrm{C}), 74.9\left(\mathrm{CH}_{2}\right), 66.3(\mathrm{CH}), 56.6(\mathrm{CH}), 47.8(\mathrm{CH}), 43.4(\mathrm{C}), 40.5(\mathrm{C}), 39.3$ $\left(\mathrm{CH}_{2}\right), 35.1(\mathrm{C}), 35.0\left(\mathrm{CH}_{2}\right), 33.6(\mathrm{CH}), 33.3\left(\mathrm{CH}_{2}\right), 32.9\left(\mathrm{CH}_{2}\right), 21.1\left(\mathrm{CH}_{3}\right), 19.3\left(\mathrm{CH}_{3}\right), 16.6(2$ x $\left.\mathrm{CH}_{3}\right), 13.5\left(\mathrm{CH}_{3}\right), 13.1\left(\mathrm{CH}_{2}\right)$. EI-MS, m/z (\%) 581 (37); 566 (3); 427 (19); 409 (36); 135 (100).

Oxidation with 3-chloroperoxybenzoic acid. To a stirred solution of a compound 4 (91 $\mathrm{mg}$; $0.22 \mathrm{mmol}$ ) in dichloromethane $(5 \mathrm{~mL}) 3$-chloroperoxybenzoic acid (43 $\mathrm{mg}$ of a commercial 50$60 \%$ MCPBA; $\sim 0.19 \mathrm{mmol}$ ) was added. The reaction mixture was stirred at room temperature for $1 \mathrm{~h}$, quenched by addition of aqueous solution of sodium sulfide and extracted with chloroform. The extract was dried over anhydrous magnesium sulfate and the solvent was evaporated in vacuo. The reaction products were separated by silica gel column chromatography. 6 $\beta$-Methoxy-3 $\alpha, 5$-cyclo-5 $\alpha$-furost-22-en-20-ol (6). Eluted with 10\% ethyl acetate/hexane as an amorphous solid, yield $28 \mathrm{mg}$ (30\%). IR, $v_{\max } 3589,3437,1690,1096 \mathrm{~cm}^{-1} .{ }^{1} \mathrm{H}$ NMR, $\delta 4.91$ $(1 \mathrm{H}, \mathrm{m}, 16 \alpha-\mathrm{H}), 4.33\left(1 \mathrm{H}, \mathrm{dd}, J_{1}=8.1 \mathrm{~Hz}, J_{2}=6.6 \mathrm{~Hz}, 23-\mathrm{H}\right), 3.32\left(3 \mathrm{H}, \mathrm{s}, \mathrm{OCH}_{3}\right), 2.78(1 \mathrm{H}, \mathrm{m}$, $6 \alpha-\mathrm{H}), 1.51(3 \mathrm{H}, \mathrm{s}, 21-\mathrm{H}), 1.02(3 \mathrm{H}, \mathrm{s}, 19-\mathrm{H}), 0.88$ and $0.86(6 \mathrm{H}, 2 \mathrm{x} \mathrm{d}, J=6.5 \mathrm{~Hz}, 26-$ and $27-$ $\mathrm{H}), 0.85(3 \mathrm{H}, \mathrm{s}, 18-\mathrm{H}), 0.65(1 \mathrm{H}, \mathrm{m}, 4-\mathrm{H}), 0.44\left(1 \mathrm{H}, \mathrm{dd}, J_{1}=8.0 \mathrm{~Hz}, J_{2}=5.1 \mathrm{~Hz}, 4-\mathrm{H}\right) .{ }^{13} \mathrm{C}$ NMR, $\delta 161.9(\mathrm{C}), 93.2(\mathrm{CH}), 83.9(\mathrm{CH}), 82.1(\mathrm{CH}), 77.6(\mathrm{C}), 66.3(\mathrm{CH}), 56.6\left(\mathrm{CH}_{3}\right), 56.6$ $(\mathrm{CH}), 47.8(\mathrm{CH}), 43.4(\mathrm{C}), 40.6(\mathrm{C}), 39.5\left(\mathrm{CH}_{2}\right), 35.2(\mathrm{C}), 35.0\left(\mathrm{CH}_{2}\right), 34.0\left(\mathrm{CH}_{2}\right), 33.3\left(\mathrm{CH}_{2}\right)$, $33.1\left(\mathrm{CH}_{2}\right), 29.6(\mathrm{CH}), 28.8(\mathrm{CH}), 24.9\left(\mathrm{CH}_{2}\right), 22.5\left(\mathrm{CH}_{3}\right), 22.3\left(\mathrm{CH}_{3}\right), 21.9\left(\mathrm{CH}_{2}\right), 21.5(\mathrm{CH})$, $21.2\left(\mathrm{CH}_{3}\right), 19.3\left(\mathrm{CH}_{3}\right), 13.5\left(\mathrm{CH}_{3}\right), 13.1\left(\mathrm{CH}_{2}\right)$. EI-MS, $\mathrm{m} / \mathrm{z}(\%) 428\left(\mathrm{M}^{+}, 27\right), 385(70), 335$ (12), 253 (37), 121 (100). HR-MS for $\mathrm{C}_{28} \mathrm{H}_{44} \mathrm{O}_{3}$, found: 428.3294; calculated: 428.3291.

6 $\beta$-Methoxy-3 $\alpha, 5$-cyclo-5 $\alpha$-furost-20(22)-en-23-one (7). Eluted with $4 \%$ ethyl acetate/hexane as an oil, yield $16 \mathrm{mg}(17 \%)$. IR, $v_{\max } 1683,1615,1091 \mathrm{~cm}^{-1} .{ }^{1} \mathrm{H} \mathrm{NMR}, \delta 4.83(1 \mathrm{H}, \mathrm{m}, 16 \alpha-\mathrm{H})$, $3.33\left(3 \mathrm{H}, \mathrm{s}, \mathrm{OCH}_{3}\right), 2.79(1 \mathrm{H}, \mathrm{m}, 6 \alpha-\mathrm{H}), 2.62\left(1 \mathrm{H}, \mathrm{dd}, J_{1}=10.3 \mathrm{~Hz}, J_{2}=1.1 \mathrm{~Hz}, 17 \alpha-\mathrm{H}\right), 2.43$ $\left(2 \mathrm{H}, \mathrm{dd}, J_{1}=7.0 \mathrm{~Hz}, J_{2}=5.4 \mathrm{~Hz}, 24-\mathrm{H}\right), 2.02(3 \mathrm{H}, \mathrm{d}, J=1.1 \mathrm{~Hz}, 21-\mathrm{H}), 1.04(3 \mathrm{H}, \mathrm{s}, 19-\mathrm{H}), 0.93$ and $0.92(2 \times \mathrm{d}, 6 \mathrm{H}, J(25,26)=J(25,27)=6.6 \mathrm{~Hz}, 3 \times \mathrm{H}-26$ and $3 \times \mathrm{H}-27) ; 0.77(\mathrm{~s}, 3 \mathrm{H}, 3 \times \mathrm{H}-$ 18); $0.67(1 \mathrm{H}, \mathrm{m}, 4-\mathrm{H}), 0.45\left(1 \mathrm{H}, \mathrm{dd}, J_{1}=8.0 \mathrm{~Hz}, J_{2}=5.2 \mathrm{~Hz}, 4-\mathrm{H}\right) .{ }^{13} \mathrm{C}$ NMR, $\delta 196.6(\mathrm{C})$, $148.5(\mathrm{C}), 123.1(\mathrm{C}), 84.4(\mathrm{CH}), 82.1(\mathrm{CH}), 65.6(\mathrm{CH}), 56.6\left(\mathrm{CH}_{3}\right), 54.8(\mathrm{CH}), 49.3\left(\mathrm{CH}_{2}\right), 47.9$ $(\mathrm{CH}), 44.7(\mathrm{C}), 43.4(\mathrm{C}), 39.9\left(\mathrm{CH}_{2}\right), 35.4\left(\mathrm{CH}_{2}\right), 35.2(\mathrm{C}), 34.3\left(\mathrm{CH}_{2}\right), 33.3\left(\mathrm{CH}_{2}\right), 29.9(\mathrm{CH})$, $24.9\left(\mathrm{CH}_{2}\right), 24.3(\mathrm{CH}), 22.7\left(\mathrm{CH}_{3}\right), 22.6\left(\mathrm{CH}_{2}\right), 22.6\left(\mathrm{CH}_{3}\right), 21.4(\mathrm{CH}), 19.3\left(\mathrm{CH}_{3}\right), 14.3(2 \mathrm{x}$ 
$\left.\mathrm{CH}_{3}\right), 13.2\left(\mathrm{CH}_{2}\right)$. EI-MS, m/z (\%) $426\left(\mathrm{M}^{+}, 32\right), 394\left(\mathrm{M}^{+}-\mathrm{MeOH}, 18\right), 180$ (100). HR-MS for $\mathrm{C}_{28} \mathrm{H}_{42} \mathrm{O}_{3}$, found: 426.3138 ; calculated: 426.3134 .

$20 \alpha$-Hydroxy-6 $\beta$-methoxy-3 $\alpha, 5$-cyclo-5 $\alpha$-pregnane-20,16 $\beta$-carbolactone (8). Eluted with $20 \%$ ethyl acetate/hexane as an amorphous solid, yield 22\% (achieved when additional $43 \mathrm{mg}$ of MCPBA was added and the reaction time was extended to 2 days). IR, $v_{\max } 3575,3347,1766$, $1097 \mathrm{~cm}^{-1} .{ }^{1} \mathrm{H}$ NMR, $\delta 5.09(1 \mathrm{H}, \mathrm{m}, 16 \alpha-\mathrm{H}), 3.34\left(3 \mathrm{H}, \mathrm{s}, \mathrm{OCH}_{3}\right), 2.79(1 \mathrm{H}, \mathrm{m}, 6 \alpha-\mathrm{H}), 2.27(1 \mathrm{H}$, m), $2.11(1 \mathrm{H}, \mathrm{d}, J=8.6 \mathrm{~Hz}, 17 \alpha-\mathrm{H}), 1.59(3 \mathrm{H}, \mathrm{s}, 21-\mathrm{H}), 1.03(3 \mathrm{H}, \mathrm{s}, 19-\mathrm{H}), 0.87$ (3H, s, 18-H), $0.67(1 \mathrm{H}, \mathrm{m}, 4-\mathrm{H}), 0.47\left(1 \mathrm{H}, \mathrm{dd}, J_{1}=8.0 \mathrm{~Hz}, J_{2}=5.2 \mathrm{~Hz}, 4-\mathrm{H}\right)$. EI-MS, $m / z(\%) 374\left(\mathrm{M}^{+}, 18\right)$, $359\left(\mathrm{M}^{+}-\mathrm{CH}_{3}, 21\right), 342\left(\mathrm{M}^{+}-\mathrm{MeOH}, 47\right), 319$ (100). HR-MS for $\mathrm{C}_{23} \mathrm{H}_{34} \mathrm{O}_{4}$, found: 374.2464 ; calculated: 374.2457.

Oxidation with 3-chloroperoxybenzoic acid in the buffered medium. To a stirred solution of compound 3 (90 $\mathrm{mg}, 0.16 \mathrm{mmol})$ in dichloromethane $(10 \mathrm{~mL})$, a $9 \%$ solution of sodium hydrogen carbonate in water $(3 \mathrm{~mL})$ and a solution of 3-chloroperoxybenzoic acid ( $80 \mathrm{mg}$ of a commercial 50-60\% MCPBA; $\sim 0.35 \mathrm{mmol}$ ) in dichloromethane $(2 \mathrm{~mL}$ ) were added. The reaction mixture was stirred for 3 days at room temperature, quenched by addition of aqueous solution of sodium sulfide, and extracted with chloroform. The extract was dried over anhydrous magnesium sulfate and the solvent was evaporated in vacuo. The products were separated by silica gel column chromatography.

$20 \alpha$-Hydroxy-6 $\beta$-methoxy-3 $\alpha, 5$-cyclo-5 $\alpha$-pregnane-20,16 $\beta$-carbolactone (8). Eluted with $18 \%$ ethyl acetate/hexane as an amorphous solid, yield $6 \mathrm{mg}(9 \%)$. Identical in all respects with compound $\mathbf{8}$ isolated from the previous reaction.

16 $\beta$-Hydroxy-6 $\beta$-methoxy-20-oxo-3 $\alpha, 5$-cyclo-5 $\alpha$-pregnanyl 4 '-methyltetrahydro-2'-furoate (9). Eluted with $20 \%$ ethyl acetate/hexane as an amorphous solid, yield $13 \mathrm{mg}(18 \%) . \mathrm{IR}, v_{\max }$ 1733, 1718, 1263, $1098 \mathrm{~cm}^{-1} .{ }^{1} \mathrm{H}$ NMR, $\delta 5.59(1 \mathrm{H}, \mathrm{m}, 16 \alpha-\mathrm{H}), 4.39$ (1H, t, $\left.J=8.0 \mathrm{~Hz}, 2^{\prime}-\mathrm{H}\right)$, $4.02\left(1 \mathrm{H}, \mathrm{m}, 5^{\prime}-\mathrm{H}\right), 3.47\left(1 \mathrm{H}, \mathrm{t}, J=8.5 \mathrm{~Hz}, 5^{\prime}-\mathrm{H}\right), 3.34\left(3 \mathrm{H}, \mathrm{s}, \mathrm{OCH}_{3}\right), 2.78(1 \mathrm{H}, \mathrm{m}, 6 \alpha-\mathrm{H}), 2.08$ $(3 \mathrm{H}, \mathrm{s}, 21-\mathrm{H}), 1.09$ (3H, s, 18-H), 1.06 (3H, d, J = 6.8 Hz, 4'-CH, 1.04 (3H, s, 19-H), 0.67 (1H, $\mathrm{m}, 4-\mathrm{H}), 0.46\left(1 \mathrm{H}, \mathrm{dd}, J_{1}=8.0 \mathrm{~Hz}, J_{2}=5.2 \mathrm{~Hz}, 4-\mathrm{H}\right) .{ }^{13} \mathrm{C} \mathrm{NMR}, \delta 205.2(\mathrm{C}), 172.9(\mathrm{C}), 82.0$ $(\mathrm{CH}), 76.9(\mathrm{CH}), 75.9\left(\mathrm{CH}_{2}\right), 74.9(\mathrm{CH}), 66.9(\mathrm{CH}), 56.8\left(\mathrm{CH}_{3}\right), 54.1(\mathrm{CH}), 48.1(\mathrm{CH}), 43.5(\mathrm{C})$, $42.6(\mathrm{C}), 38.4\left(\mathrm{CH}_{2}\right), 35.2\left(\mathrm{CH}_{2}\right), 35.1\left(2 \mathrm{x} \mathrm{CH}_{2}\right), 34.2(\mathrm{CH}), 33.3\left(\mathrm{CH}_{2}\right), 30.5\left(\mathrm{CH}_{3}\right), 29.6(\mathrm{C})$, $29.5(\mathrm{CH}), 24.9\left(\mathrm{CH}_{2}\right), 21.9\left(\mathrm{CH}_{2}\right), 21.3(\mathrm{CH}), 19.3\left(\mathrm{CH}_{3}\right), 16.1\left(\mathrm{CH}_{3}\right), 13.7\left(\mathrm{CH}_{3}\right), 13.2\left(\mathrm{CH}_{2}\right)$. EI-MS, m/z (\%) $458\left(\mathrm{M}^{+}, 8\right), 443\left(\mathrm{M}^{+}-\mathrm{CH}_{3}, 31\right), 426\left(\mathrm{M}^{+}-\mathrm{MeOH}, 23\right), 403$ (56), 328 (14), 296 (49), 85 (100). HR-MS for $\mathrm{C}_{28} \mathrm{H}_{42} \mathrm{O}_{5}$, found: 458.3051 ; calculated: 458.3032.

\section{6 $\beta$-Hydroxy-6 $\beta$-methoxy-20-oxo-3 $\alpha, 5$-cyclo-5 $\alpha$-pregnanyl 2'-hydroxy-5'-tosyloxy-4'-}

methyl-pentanoate (10). Eluted with $40 \%$ ethyl acetate/hexane as an amorphous solid, yield 50 mg (51\%). IR, $v_{\max } 3542,1732,1720,1374,1179,1099 \mathrm{~cm}^{-1} .{ }^{1} \mathrm{H} \mathrm{NMR}, \delta 7.79(2 \mathrm{H}, \mathrm{d}, J=8.3$

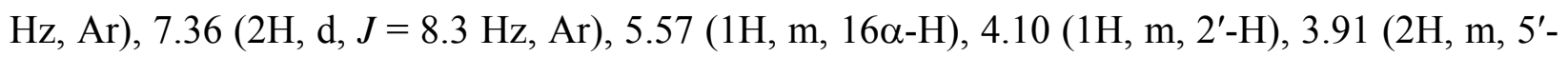
$\mathrm{H}), 3.34\left(3 \mathrm{H}, \mathrm{s}, \mathrm{OCH}_{3}\right), 2.79(1 \mathrm{H}, \mathrm{m}, 6 \alpha-\mathrm{H}), 2.46\left(3 \mathrm{H}, \mathrm{s}, \mathrm{Ar}-\mathrm{CH}_{3}\right), 2.08(3 \mathrm{H}, \mathrm{s}, 21-\mathrm{H}), 1.06(3 \mathrm{H}$, s, 18-H), $1.03(3 \mathrm{H}, \mathrm{s}, 19-\mathrm{H}), 0.97\left(3 \mathrm{H}, \mathrm{d}, J=6.7 \mathrm{~Hz}, 4^{\prime}-\mathrm{CH}_{3}\right), 0.68$ (1H, m, 4-H), 0.47 (1H, dd, $\left.J_{1}=8.0 \mathrm{~Hz}, J_{2}=5.0 \mathrm{~Hz}, 4-\mathrm{H}\right)$. 
Oxidation with osmium tetroxide. To a stirred solution of compound 4 (58 $\mathrm{mg}, 0.14 \mathrm{mmol}$ ) in pyridine $(3 \mathrm{ml})$, a solution of osmium tetroxide $(38 \mathrm{mg}, 0.15 \mathrm{mmol})$ in pyridine $(2 \mathrm{~mL})$ was added. The reaction mixture was stirred at room temperature for $18 \mathrm{~h}$, additional $6 \mathrm{~mL}$ of pyridine, $8 \mathrm{~mL}$ of water, and aqueous solution of sodium hydrogen sulfite $(2 \mathrm{~mL}$ of $40 \%$ $\mathrm{NaHSO}_{3}$ ) were added in order to reduce osmium complex. The reaction mixture was stirred for 2 days, poured into water and extracted with ether. The extract was washed with water, the solvent was removed in vacuo from the extract, and the crude product was purified by silica gel chromatography. 16 $\beta, 20 \alpha$-Dihydroxy-6 $\beta$-methoxy-3 $\alpha, 5$-cyclo-5 $\alpha$-cholestan-22-one (11) was eluted with $25 \%$ ethyl acetate/hexane, yield $30 \mathrm{mg}(49 \%)$, m.p. $179-182^{\circ} \mathrm{C}$ (dichloromethane hexane). IR, $v_{\max } 3689,3446,1702,1093 \mathrm{~cm}^{-1} .{ }^{1} \mathrm{H} \mathrm{NMR}, \delta 4.71(1 \mathrm{H}, \mathrm{m}, 16 \alpha-\mathrm{H}), 4.43(1 \mathrm{H}, \mathrm{s}$, $\mathrm{OH}), 3.87(1 \mathrm{H}, \mathrm{brs}, \mathrm{OH}), 3.32\left(3 \mathrm{H}, \mathrm{s}, \mathrm{OCH}_{3}\right), 2.77(1 \mathrm{H}, \mathrm{m}, 6 \alpha-\mathrm{H}), 2.65(2 \mathrm{H}, \mathrm{t}, J=7.5 \mathrm{~Hz}, 23-$ H), $2.30(1 \mathrm{H}, \mathrm{m}), 1.56(3 \mathrm{H}, \mathrm{s}, 21-\mathrm{H}), 1.05(3 \mathrm{H}, \mathrm{s}, 18-\mathrm{H}), 1.02(3 \mathrm{H}, \mathrm{s}, 19-\mathrm{H}), 0.93(6 \mathrm{H}, \mathrm{d}, J=6.1$ $\mathrm{Hz}, 26-$ and $27-\mathrm{H}), 0.65(1 \mathrm{H}, \mathrm{m}, 4-\mathrm{H}) ; 0.44\left(1 \mathrm{H}, \mathrm{dd}, J_{1}=8.0 \mathrm{~Hz}, J_{2}=5.1 \mathrm{~Hz}, 4-\mathrm{H}\right) .{ }^{13} \mathrm{C} \mathrm{NMR}, \delta$ $214.5(\mathrm{C}), 82.1(\mathrm{CH}), 81.5(\mathrm{C}), 73.5(\mathrm{CH}), 59.6(\mathrm{CH}), 56.5\left(\mathrm{CH}_{3}\right), 54.4(\mathrm{CH}), 48.0(\mathrm{CH}), 43.4$ (C), $42.6(\mathrm{C}), 39.2\left(\mathrm{CH}_{2}\right), 37.1\left(\mathrm{CH}_{2}\right), 35.3(\mathrm{C}) ; 35.2\left(\mathrm{CH}_{2}\right) ; 34.8\left(\mathrm{CH}_{2}\right) ; 33.3\left(\mathrm{CH}_{2}\right) ; 32.4\left(\mathrm{CH}_{2}\right)$; $29.4(\mathrm{CH}) ; 27.7(\mathrm{CH}) ; 26.7\left(\mathrm{CH}_{3}\right) ; 24.9\left(\mathrm{CH}_{2}\right), 22.4\left(2 \times \mathrm{CH}_{3}\right), 22.1\left(\mathrm{CH}_{2}\right), 21.5(\mathrm{CH}), 19.3$ $\left(\mathrm{CH}_{3}\right), 15.2\left(\mathrm{CH}_{3}\right), 13.0\left(\mathrm{CH}_{2}\right)$. EI-MS, m/z (\%) $428\left(\mathrm{M}^{+}-\mathrm{H}_{2} \mathrm{O}, 12\right), 385\left(\mathrm{M}^{+}-\mathrm{C}_{3} \mathrm{H}_{7}, 30\right), 347$ (100). HR-MS (ESI) for $\mathrm{C}_{28} \mathrm{H}_{46} \mathrm{O}_{4} \mathrm{Na}$, found 469.3288; calculated: 469.3294 .

Oxidation with osmium tetroxide in the presence of $\boldsymbol{N}$-methylmorpholine $\boldsymbol{N}$-oxide. To a stirred solution of compound $3(60 \mathrm{mg}, 0.1 \mathrm{mmol})$ in acetone $(3 \mathrm{~mL})$, NMO (140 $\mathrm{mg}, 0.12$ $\mathrm{mmol}$ ) and three drops of $10 \%$ solution of $\mathrm{OsO}_{4}$ in benzene were added. The reaction mixture was stirred at room temperature for 3 days, then an aqueous solution of sodium hydrogen sulfite ( $2 \mathrm{~mL}, 40 \%$ ) was added. The reaction mixture was stirred at room temperature overnight, poured into water and extracted with ether. The extract was dried over anhydrous magnesium sulfate and the solvent was evaporated in vacuo. The residue was purified by silica gel chromatography. Elution with $30 \%$ ethyl acetate/hexane afforded $16 \beta, 20 \alpha, 26$-trihydroxy-6 $\beta$-methoxy-3 $\alpha, 5$-cyclo$5 \alpha$-cholestan-22-one 26-tosylate (12) as an amorphous solid (28 mg, 45\%). IR, $v_{\max } 3684,3469$, 1601, $1176 \mathrm{~cm}^{-1} .{ }^{1} \mathrm{H}$ NMR, $\delta 7.79(2 \mathrm{H}, \mathrm{d}, J=8.2 \mathrm{~Hz}, \mathrm{Ar}), 7.35$ (2H, d, J=8.2 Hz, Ar), 4.69 (1H, m, 16 $\alpha-\mathrm{H}), 4.40(1 \mathrm{H}, \mathrm{brs}, \mathrm{OH}), 3.88(2 \mathrm{H}, \mathrm{dd}, 26-\mathrm{H}), 3.32\left(3 \mathrm{H}, \mathrm{s}, \mathrm{OCH}_{3}\right), 2.77(1 \mathrm{H}, \mathrm{m}, 6 \alpha-\mathrm{H})$, $2.69(2 \mathrm{H}, \mathrm{m}, 23-\mathrm{H}), 2.45\left(3 \mathrm{H}, \mathrm{s}, \mathrm{Ar}-\mathrm{CH}_{3}\right), 1.49(3 \mathrm{H}, \mathrm{s}, 21-\mathrm{H}), 1.04(3 \mathrm{H}, \mathrm{s}, 19-\mathrm{H}), 0.89$ (3H, s, $18-\mathrm{H}), 0.93(3 \mathrm{H}, \mathrm{d}, J=6.7 \mathrm{~Hz}, 27-\mathrm{H}), 0.65(1 \mathrm{H}, \mathrm{m}, 4-\mathrm{H}), 0.44\left(1 \mathrm{H}, \mathrm{dd}, J_{1}=7.9 \mathrm{~Hz}, J_{2}=5.2 \mathrm{~Hz}\right.$, 4-H).

Oxidation with osmium tetroxide in the presence of hydrogen peroxide. To a stirred solution of compound 3 (111 mg, $0.19 \mathrm{mmol})$ in tert-butanol $(25 \mathrm{~mL})$, an aqueous solution of hydrogen peroxide $(30 \%, 5 \mathrm{~mL})$ and three drops of $10 \%$ solution of $\mathrm{OsO}_{4}$ in benzene were added. The reaction mixture was stirred at room temperature for 3 days and an aqueous solution of sodium hydrogen sulfite $(40 \%, 2 \mathrm{~mL})$ was then added. The reaction mixture was stirred at room temperature overnight, poured into water and extracted with ether. The extract was dried over anhydrous magnesium sulfate and the solvent was evaporated in vacuo. The crude product was

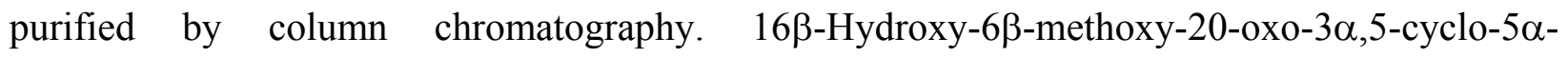


pregnanyl 5'-tosyloxy-4'-methylpentanoate (13) was eluted (52 mg, 45\%) with 25\% ethyl acetate/hexane as an amorphous solid. IR, $v_{\max } 1732,1713,1359,1176 \mathrm{~cm}^{-1} .{ }^{1} \mathrm{H}$ NMR, 7.79 $(2 \mathrm{H}, \mathrm{d}, J=8.2 \mathrm{~Hz}, \mathrm{Ar}), 7.35(2 \mathrm{H}, \mathrm{d}, J=8.2 \mathrm{~Hz}, \mathrm{Ar}), 5.48(1 \mathrm{H}, \mathrm{m}, 16-\mathrm{H}), 3.85$ (2H, m, 26-H), $3.32\left(3 \mathrm{H}, \mathrm{s}, \mathrm{OCH}_{3}\right), 2.77(1 \mathrm{H}, \mathrm{m}, 6 \alpha-\mathrm{H}), 2.46\left(3 \mathrm{H}, \mathrm{s}, \mathrm{Ar}-\mathrm{CH}_{3}\right), 2.40(2 \mathrm{H}, \mathrm{m}, 23-\mathrm{H}), 2.06(3 \mathrm{H}, \mathrm{s}$, 21-H), 1.06 (3H, s, 18-H), 1.04 (3H, s, 19-H), 0.90 (3H, d, J=6.7 Hz, 27-H), 0.66 (1H, m, 4-H), $0.45\left(1 \mathrm{H}, \mathrm{dd}, J_{1}=8.0 \mathrm{~Hz}, J_{2}=5.2 \mathrm{~Hz}, 4-\mathrm{H}\right) .{ }^{13} \mathrm{C} \mathrm{NMR}, \quad 205.4(\mathrm{C}), 172.6(\mathrm{C}), 144.7(\mathrm{C}), 132.9$ (C), $129.8(\mathrm{CH}), 128.2(\mathrm{CH}), 127.8(2 \times \mathrm{CH}), 81.9(\mathrm{CH}), 74.4(\mathrm{CH}), 74.2\left(\mathrm{CH}_{2}\right), 66.6(\mathrm{CH}), 56.6$ $\left(\mathrm{CH}_{3}\right), 53.9(\mathrm{CH}), 48.1(\mathrm{CH}), 43.4(\mathrm{C}), 42.7(\mathrm{C}), 38.4\left(\mathrm{CH}_{2}\right), 35.1(\mathrm{C}), 34.9\left(2 \times \mathrm{CH}_{2}\right), 33.2$ $\left(\mathrm{CH}_{2}\right), 32.2\left(\mathrm{CH}_{2}\right), 31.5\left(\mathrm{CH}_{2}\right), 30.7\left(\mathrm{CH}_{3}\right), 27.5\left(\mathrm{CH}_{2}\right), 24.8\left(\mathrm{CH}_{2}\right), 21.9\left(\mathrm{CH}_{2}\right), 21.5(\mathrm{CH}), 21.3$ (CH), $21.3\left(\mathrm{CH}_{3}\right), 19.2\left(\mathrm{CH}_{3}\right), 15.9\left(\mathrm{CH}_{3}\right), 13.7\left(\mathrm{CH}_{3}\right), 12.98\left(\mathrm{CH}_{2}\right)$. EI-MS, m/z $(\%) 614\left(\mathrm{M}^{+}\right.$, 7), 599 (34), 582 (10), 559 (32), 296 (81), 43 (100).

\section{Acknowledgments}

Financial support from the Polish Ministry of Education is gratefully acknowledged. We also thank Mrs. J. Maj for help in preparation of the manuscript.

\section{References}

1. Hostettmann, K.; Marston, A. Saponins, Cambridge University Press: Cambridge, UK, 1995.

2. Torgov, I. V. Ed Khimiya Spirostanolov, Akad. Nauk SSSR: Moscow, 1986.

3. Fieser, L.; Fieser, M. Steroids, Reinhold Publishing Corporation: New York, 1959.

4. Raber, L. Chem. Eng. News 1999, October 25, 78.

5. Marker, R. E.; Wagner, R. B.; Ulshafer, P. R.; Wittbecker, E. L.; Goldsmith, D. P. J.; Ruof, C. H. J. Am. Chem. Soc. 1947, 69, 21.

6. Lemieux, R. U.; von Rudolff, E. Can. J. Chem. 1955, 33, 1710.

7. Ruan, B.; Wilson, W. K.; Schroepfer Jr., G. J. Steroids 1999, 64, 385.

8. Dong, M.; Feng, X.; Wang, B.; Wu, L.; Ikejima, T. Tetrahedron 2001, 57, 501.

9. Garrido, L.; Zubia, E.; Ortega, M. J.; Salvă, J. Steroids 2000, 65, 85.

10. Morzycki, J. W.; Jastrzębska, I.; Katryński, K. Collect. Czech. Chem. Commun. 2001, 66, 1746.

11. Ishikawa, K.; Charles, H. C.; Griffin, G. W. Tetrahedron Lett. 1977, 427.

12. Murray, R. W. Chem. Rev. 1989, 89, 1187.

13. McCormick, J. P.; Tomasik, W.; Johnson, M. W. Tetrahedron Lett. 1981, 22, 607. 